\title{
Behavior of Polyethylene Glycol Molecules at an Oscillating Solid-Liquid Interface
}

\author{
Minoru Yoshimoto $^{1 *}$, Yukiko Yuda ${ }^{1}$, Mutsuo Tanaka $^{2}$ and Shigeru Kurosawa ${ }^{3}$ \\ ${ }^{1}$ Department of Information Science and Biomedical Engineering, Graduate School of Science and Engineering, Kagoshima University, 1-21-40 \\ Korimoto, Kagoshima 890-0065, Japan \\ ${ }^{2}$ Biomedical Research Institute, National Institute of Advanced Industrial Science and Technology (AIST), Central 6, 1-1-1 Higashi, Tsukuba, \\ Ibaraki 305-8566, JAPAN \\ ${ }^{3}$ Research Institute for Environmental Management Technology, National Institute of Advanced Industrial Science and Technology (AIST), AIST \\ Tsukuba West, 16-1 Onogawa, Tsukuba, Ibaraki 305-8569, Japan
}

\begin{abstract}
The behavior of polyethylene glycol (PEG) molecules at an oscillating solid-liquid interface was examined using a quartz crystal microbalance (QCM). The number-average molecular weights $\left(M_{n}\right)$ of $P E G$ molecules were systematically varied. This study revealed that the shift in the energy dissipation factor, $\Delta D$, of the QCM against square root of the density-viscosity product of the PEG solution is linear and has an intercept. Moreover, the systematical analysis revealed that the $\Delta D$ slope rapidly decreases with $M_{\mathrm{n}}$, and the $\Delta D$ intercept becomes constant above $1.1 \times 10^{4} \mathrm{~g} / \mathrm{mol}$. Those results indicated that the resonant length of PEG molecules moving with the oscillating plate at $9 \mathrm{MHz}$ is $97.6 \AA$. We also found that the difference between the resonant lengths calculated from $\Delta D$ and series resonance-frequency shift, $\Delta F$, is related to the density of the thin PEG layer formed on the gold electrode.
\end{abstract}

Key words: QCM, solid-liquid interface, polyethylene glycol, energy dissipation, Debye process

\section{INTRODUCTION}

For a long time, a quartz crystal microbalance (QCM) has been used in vacuum and gaseous environments as a mass sensor $^{1-3)}$. However, its oscillation in a liquid environment was later successful, and there have recently been many requirements for sensors, which can be used in a liquid ${ }^{4)}$. They have several advantages such as ease of use, low detection limit and continuous operation that have attracted increased interest. The QCM was also applied to the Belousov-Zhabotinsky (BZ) reaction, which is known as a nonlinear chemical oscillation reaction ${ }^{5)}$. This study revealed that the density and viscosity of the solution of the BZ reaction oscillated. This new finding produced novel concepts in the field of nonlinear science.

The properties of polymer solutions are of considerable interest to both theorists and experimentalists owing to their importance in many different fields ${ }^{6-15)}$. Much effort has been devoted to obtaining a comprehensive understanding of polymer molecules in dilute, semidilute and concentrated bulk solutions. Recently, many studies have focused on the physical properties of polymer molecules at a solid-liquid interface ${ }^{16-37)}$. Most of those studies are supported by development of the measurement techniques of the $\mathrm{QCM}^{16)}$.

Polyethylene glycol (PEG) molecules are the most extensively studied linear polymers, whose chains are composed of oxyethylene units. Owing to the high water solubility and low toxicity of PEG molecules, they are employed in a wide range of applications in pharmaceutical, clinical and biomedical researches ${ }^{22,23)}$. For example, PEG molecules have the ability to reduce nonspecific adsorption of proteins at a solid-liquid interface ${ }^{24-34)}$. This is very useful for label-free biosensors such as the QCM, because nonspecific adsorption of biomolecules generates problems in quantitative estimation of target protein concentration and evaluation of the binding affinity between proteins. The degree to which the nonspecific adsorption of proteins is prevented is related to the PEG length. However, previous reports did not consider the dynamic properties of PEG molecules at the solid-liquid interface oscillating at $\mathrm{MHz}$ frequencies.

The QCM measurements rely on the frequency shift and energy dissipation shift of a quartz crystal oscillating in thickness-shearing mode at $\mathrm{MHz}$ frequencies ${ }^{16)}$. This allows the QCM to be used for investigating nanoscale rheology. Therefore, understanding the dynamic properties of polymer molecules at an oscillating solid-liquid interface is

\footnotetext{
*Correspondence to: Minoru Yoshimoto, Department of Information Science and Biomedical Engineering, Graduate School of Science and Engineering, Kagoshima University, 1-21-40 Korimoto, Kagoshima 890-0065, Japan

E-mail: myoshi@ibe.kagoshima-u.ac.jp

Accepted August 6, 2013 (received for review August 2, 2013)

Journal of Oleo Science ISSN 1345-8957 print / ISSN 1347-3352 online

http://www.jstage.jst.go.jp/browse/jos/ http://mc.manusriptcentral.com/jjocs
} 
very important from the viewpoint of both theory and experiment.

In a previous report ${ }^{37)}$, we used the QCM to study the properties of PEG molecules oscillating at $\mathrm{MHz}$ frequencies at a solid-liquid interface. This study revealed that the slope of the series resonance-frequency shift against the number-average molecular weight decreases according to the Debye process. Using this method, we found that PEG of length of $54.2 \AA$ moves with the oscillating plate at $9 \mathrm{MHz}$. However, this study only focused on the series resonance-frequency shift.

In the QCM measurements, the energy dissipation shift provides information about viscoelasticity of the polymers and characterizes those properties ${ }^{16)}$. In polymer studies, the investigation of energy dissipation shift is an important parameter, which is equivalent to series resonance-frequency shift. Therefore, in this paper, we examined the dynamic properties of PEG molecules at a solid-liquid interface oscillating at $\mathrm{MHz}$ frequencies in terms of the energy dissipation shift. With the help of the QCM, we investigate the transition behavior of PEG molecules by systematically varying the number-average molecular weight. Furthermore, by comparing series resonance-frequency shift and energy dissipation shift, we discussed the PEG resonant length moving with the oscillating solid-liquid interface in regions of the thin layer and the viscous penetration depth.

\section{EXPERIMENTAL}

\subsection{Sample preparation}

Water with specific resistance of $18.2 \mathrm{M} \Omega \cdot \mathrm{cm}$ was prepared with a Milli-Q apparatus (Millipore, Tokyo, Japan). PEG solutions were prepared using PEG-200 $\left(M_{\mathrm{n}}=246 \mathrm{~g} /\right.$ mol, $\left.M_{\mathrm{w}} / M_{\mathrm{n}}=1.05\right)$, PEG-600 $\left(M_{\mathrm{n}}=576 \mathrm{~g} / \mathrm{mol}, M_{\mathrm{w}} / M_{\mathrm{n}}=\right.$ $1.04), \mathrm{PEG}-2 \mathrm{~K}\left(M_{\mathrm{n}}=1912 \mathrm{~g} / \mathrm{mol}, M_{\mathrm{w}} / M_{\mathrm{n}}=1.01\right), \mathrm{PEG}-6 \mathrm{~K}$ $\left(M_{\mathrm{n}}=8798 \mathrm{~g} / \mathrm{mol}, M_{\mathrm{w}} / M_{\mathrm{n}}=1.00\right), \mathrm{PEG}-20 \mathrm{~K}\left(M_{\mathrm{n}}=21335 \mathrm{~g} /\right.$ mol, $\left.M_{\mathrm{w}} / M_{\mathrm{n}}=1.00\right), \mathrm{PEG}-500 \mathrm{~K}\left(M_{\mathrm{n}}=4 \times 10^{5} \mathrm{~g} / \mathrm{mol}\right)$ and PEG-2M $\left(M_{\mathrm{n}}=1.75 \times 10^{6} \mathrm{~g} / \mathrm{mol}\right)$ (Wako, Japan), where $M_{\mathrm{n}}$ and $M_{\mathrm{w}}$ are the number-average molecular weight and weight-average molecular weight, respectively ${ }^{6,7)}$. The values of $M_{\mathrm{n}}$ and $M_{\mathrm{w}}$ of PEG molecules were measured using MALDI-MS (Shimazu AXIMA-CFR plus). Unfortunately, we were unable to estimate the exact values of $M_{\mathrm{n}}$ and $M_{\mathrm{w}}$ of PEG-500K and PEG-2M because they exhibited a broad distribution of molecular weight. However, the values of $M_{\mathrm{n}}$ measured using MALDI-MS were approximately equal to the average molecular weight mentioned by Wako. Therefore, we used the average molecular weight depicted by Wako as the values of $M_{n}$.

The PEG molecules were dissolved in water by a magnetic stirring bar at room temperature, and after two days, the PEG solutions were used for viscosity, density and the
QCM analyses. For comparison, Newtonian liquids were prepared using sucrose (Wako, Japan).

\subsection{Measurements of viscosity and density}

The viscosities of the PEG solutions were measured using Cannon-Ubbelohde viscometers. The viscometers were placed in a constant temperature water bath, set at $25 \pm 0.1^{\circ} \mathrm{C} .10 \mathrm{~mL}$ of the sample solutions was used for the measurements. The PEG solutions were allowed to equilibrate to the set temperature for $20 \mathrm{~min}$ prior to the viscosity measurements.

Density values of the PEG solutions were measured using a $10 \mathrm{~mL}$ pycnometer. The pycnometer was placed in a water bath kept at $25 \pm 0.1^{\circ} \mathrm{C}$ for 20 min prior to the measurements. Weights were measured by an electronic balance (Shimazu, BW-420H).

\subsection{QCM analysis}

A $9 \mathrm{MHz}$ AT-cut QCM with a pair of gold electrodes was used for all the experiments. This QCM had a mirrored surface, and the geometry of quartz crystal plate and gold electrodes was round. The quartz crystal plate and electrodes were 8 and $5 \mathrm{~mm}$ in diameter, respectively, and the geometry and size of gold electrodes were the same on both sides. The QCM was purchased from Nihon Dempa Kogyo (Tokyo, Japan).

We used an impedance analyzer (Agilent Technologies 4294A) for studying the resonant-frequency properties of the QCM. The 16047E test fixture (Agilent Technologies) was employed for direct impedance measurements (Fig. 1a). The source power was adjusted to $0.237 \mathrm{~V}$. The frequency span was set at $10 \mathrm{kHz}$. The impedance and phase data associated with 801 frequency data points centered at the frequency of minimum impedance were recorded on a personal computer. The shift in the energy dissipation factor, $\Delta D$, was calculated using admittance analysis ${ }^{38,39)}$.

The cell was $8 \mathrm{~mL}$ and had a water jacket to maintain constant temperature. One side of the QCM was sealed with a blank quartz crystal casing, maintaining it in an air environment (Fig. 1b). The QCM was mounted in level with the water surface, and the immersion depth of the QCM was set at $0.5 \mathrm{~cm}$ (Fig. 1a). The cell and QCM system were shielded by a grounded aluminum plate. The gold electrodes of the QCM were rinsed with piranha solution $\left(\mathrm{H}_{2} \mathrm{SO}_{4}: \mathrm{H}_{2} \mathrm{O}_{2}(30 \%)=3: 1\right)$ prior to impedance measurements. For measurements in PEG solutions, the cell temperature was set to $25 \pm 0.1^{\circ} \mathrm{C}$. For physical adsorption equilibrium between the PEG molecules and the gold electrodes, the QCM was immersed in the PEG solutions for 3 $\mathrm{h}$ prior to the impedance measurements. For measurements in sucrose solutions, the cell temperature was set to $20 \pm 0.1^{\circ} \mathrm{C}$, and the QCM was immersed in sucrose for 15 min prior to the measurements. PEG and sucrose solutions were stirred using a stirring bar. The stirring was stopped 


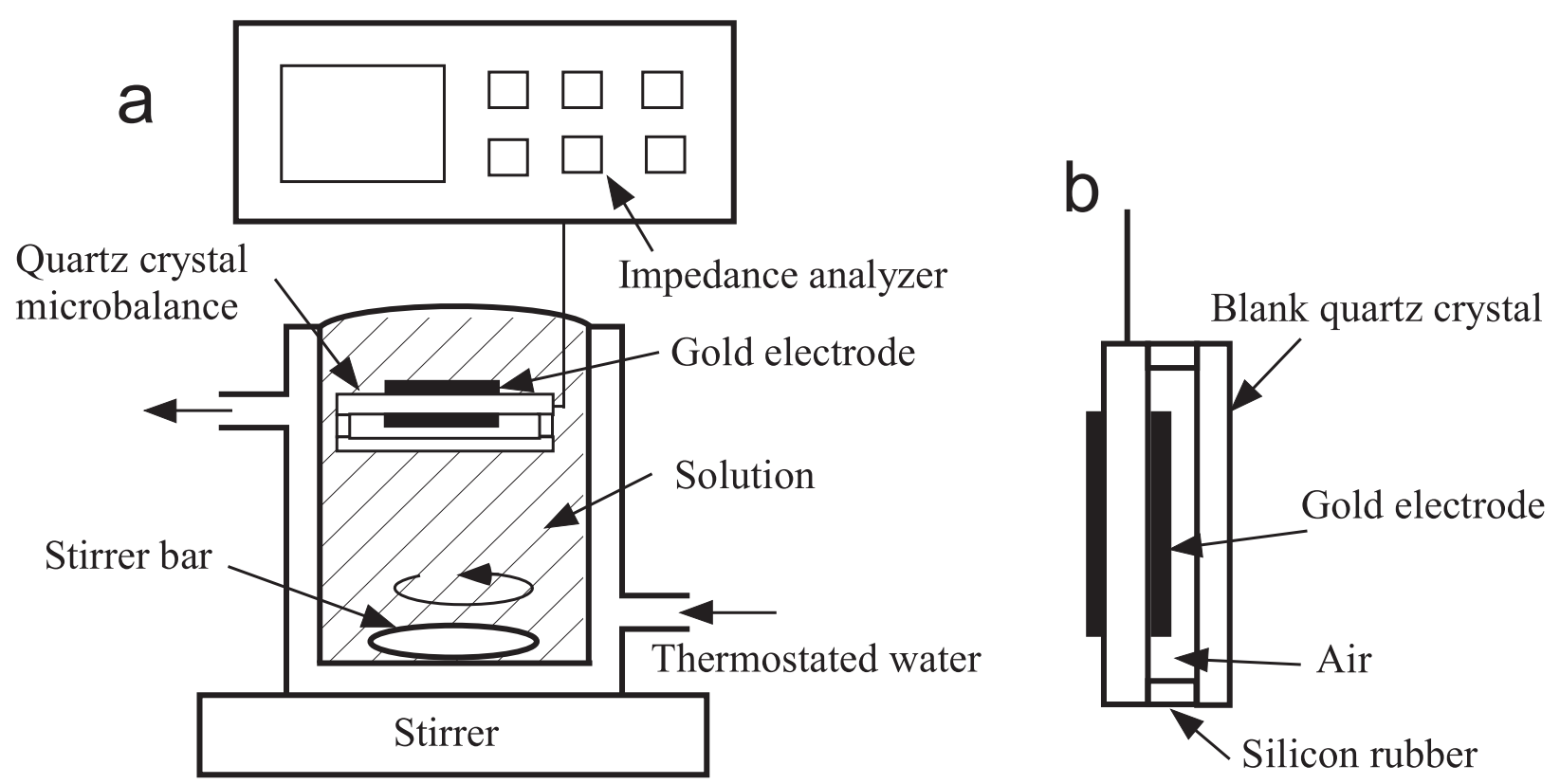

Fig. $1 a$. Schematic illustration of an experimental apparatus. $b$. Schematic illustration of the one-face sealed QCM. The cell and QCM system were shielded by a grounded aluminum plate.

during QCM measurements. The experiments were repeated to obtain statistically significant results.

\section{RESULTS}

\subsection{Behavior of $\Delta D$ in a Newtonian liquid}

Kanazawa and Gordon proposed the following equation to predict the linear relationship between series resonantfrequency shift and square root of the density-viscosity product in a Newtonian liquid ${ }^{40)}$ :

$$
\Delta f_{\eta}=-\frac{f_{q}^{3 / 2}}{\sqrt{\pi \rho_{q} \mu_{q}}} \sqrt{\rho_{1} \eta_{1}}
$$

where $\Delta f_{\eta}$ is the series resonant-frequency shift, $f_{\mathrm{q}}$ is the resonant fundamental frequency of the quartz crystal, $\mu_{\mathrm{q}}$ is the shear modulus of the quartz crystal, $\eta_{1}$ is the absolute viscosity of the Newtonian liquid, and $\rho_{\mathrm{q}}$ and $\rho_{1}$ are the densities of the quartz crystal and the Newtonian liquid, respectively. The subscripts $\mathrm{q}$ and 1 indicate the quartz crystal and Newtonian liquid, respectively. In addition, the shift in the energy dissipation factor, $\Delta D_{\eta}$, is described by ${ }^{41)}$

$$
\Delta D \eta=\frac{2 f_{q}^{1 / 2}}{\sqrt{\pi \rho_{q} \mu_{q}}} \sqrt{\rho_{1} \eta_{1}} \text {. }
$$

Figure 2 shows the experimental result for $\Delta D$ as a function of $\sqrt{\rho_{1} \eta_{1}}-\sqrt{\rho_{w} \eta_{w}}$ for the Newtonian liquid, where the subscript $\mathrm{w}$ indicates pure water. Figure 2 shows that the experimental data of $\Delta D$ vary linearly with $\sqrt{\rho_{1} \eta_{1}}-\sqrt{\rho_{w} \eta_{w}}$ and are approximately equal to the values calculated with equation (2).

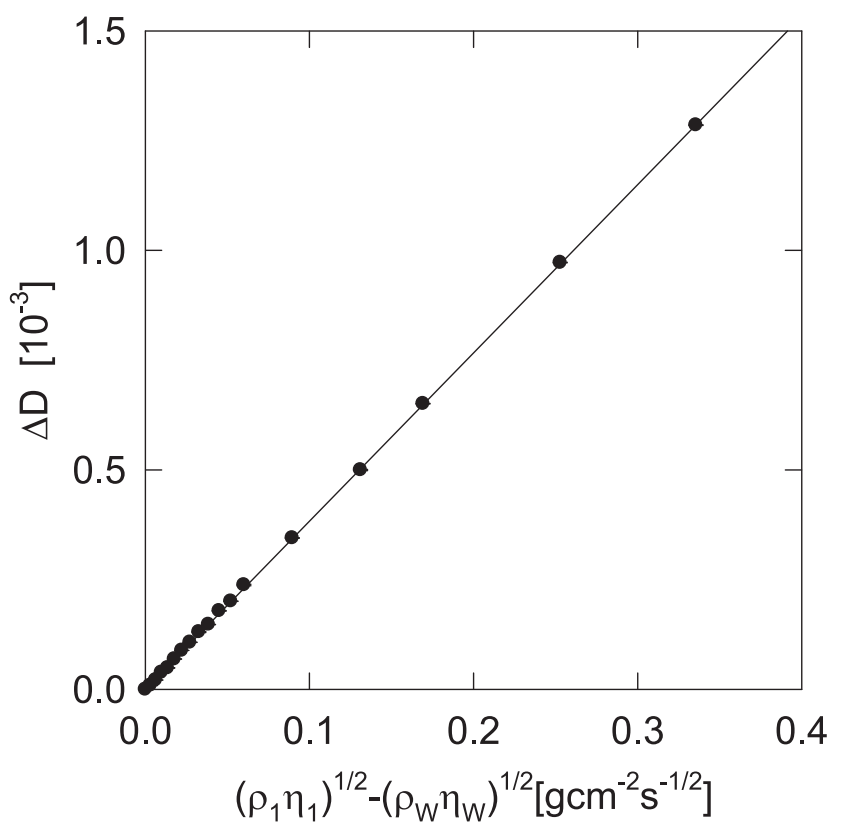

Fig. $2 \Delta D$ versus $\sqrt{\rho_{1} \eta_{1}}-\sqrt{\rho_{w} \eta_{w}}$ in sucrose solutions. The solid line means the value calculated using equation (2). The error bar represents standard deviation. Measurements were repeated six times. We employed the solution concentrations of every $2 \mathrm{wt} \%$ in $0 \sim 24 \mathrm{wt} \%$, and $30,36,40,46$, $50 \mathrm{wt} \%$.

\subsection{Behavior of $\Delta D$ in PEG solutions}

PEG solutions show properties of a non-Newtonian liquid $^{6-21,37)}$. This means that the viscosities of PEG solu- 


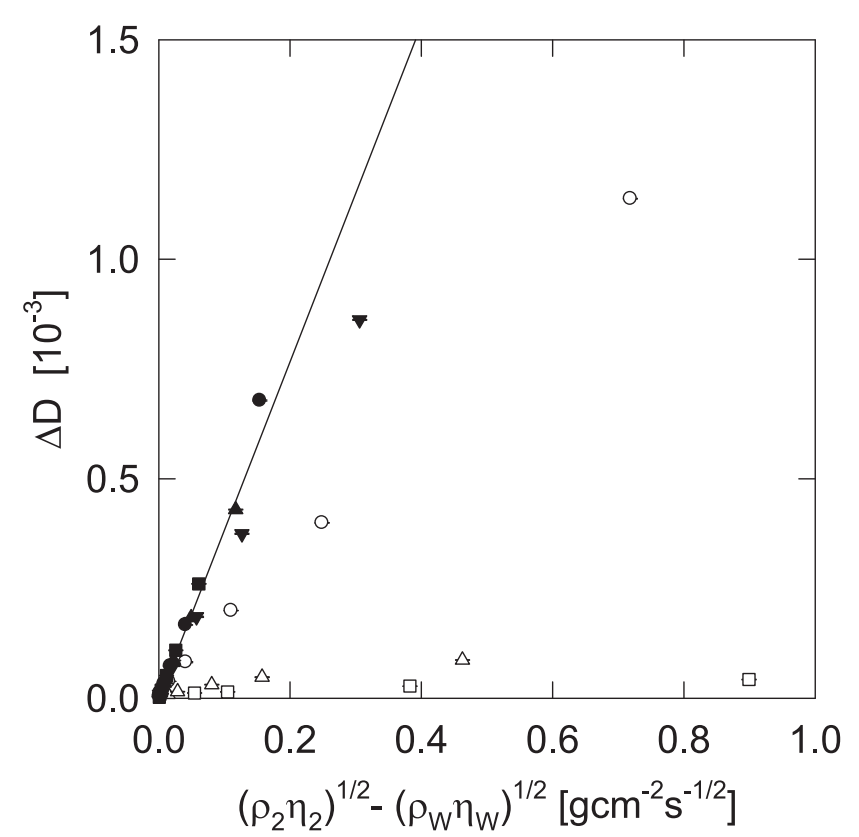

Fig. $3 \Delta D$ versus $\sqrt{\rho_{2} \eta_{2}}-\sqrt{\rho_{w} \eta_{w}}$ in PEG solutions. PEG-200, $\mathbf{\square}:$ PEG-600, $\boldsymbol{\Lambda}$ : PEG-2K, $\boldsymbol{\nabla}$ : PEG6K, $\bigcirc$ : PEG-20K, $\triangle$ : PEG-500K, $\square$ : PEG-2M. The error bar represents standard deviation. Measurements were repeated six times. The solid line was calculated using equation (2). We employed the following solution concentrations (wt\%). PEG-200: 0.5, 1, 2, 5, 10, 20, 50. PEG600: 0.5, 1, 2, 5, 10, 20. PEG-2K: 0.5, 1, 2, 5, 10. PEG-6K: 0.5, 1, 2, 5, 10, 20. PEG-20K: 0.2, 0.8, 2, 5, 10, 20. PEG-500K: 0.1, 0.2, 0.5, 0.8, 2. PEG-2M: 0.1, 0.2, 0.5, 0.8.

tions are dependent on the oscillation frequency under oscillatory shear stress. In other words, the relaxation process of PEG solutions depends on the molecular weight. Therefore, we can employ the value of $\sqrt{\rho_{2} \eta_{2}}-\sqrt{\rho_{w} \eta_{w}}$ as an index of the difference between the Newtonian liquids and PEG solutions, where the subscript 2 indicates the PEG solution. Based on this difference, we can investigate the dynamic properties of PEG molecules of varying molecular weights at the solid-liquid interface oscillating at 9MHz.

Figure 3 illustrates the results of $\Delta D$ as a function of $\sqrt{\rho_{2} \eta_{2}}-\sqrt{\rho_{w} \eta_{w}}$. For all the PEG solutions, the experimental data of $\Delta D$ vary linearly with $\sqrt{\rho_{2} \eta_{2}}-\sqrt{\rho_{w} \eta_{w}}$. The values of the $\Delta D$ slope of PEG-200, PEG-600 and PEG-2K are approximately equal to that of the Newtonian liquid. In contrast, the values of the $\Delta D$ slope of PEG-6K, PEG-20K, PEG-500K and PEG-2M are smaller than that of the Newtonian liquid.

To get further insight on the relationship between $\Delta D$ and $\sqrt{\rho_{2} \eta_{2}}-\sqrt{\rho_{w} \eta_{w}}$, we analyzed the $\Delta D$ data using a leastsquare method. A representative example is shown in Fig. 4 , where the data of PEG-6k solution are presented. The

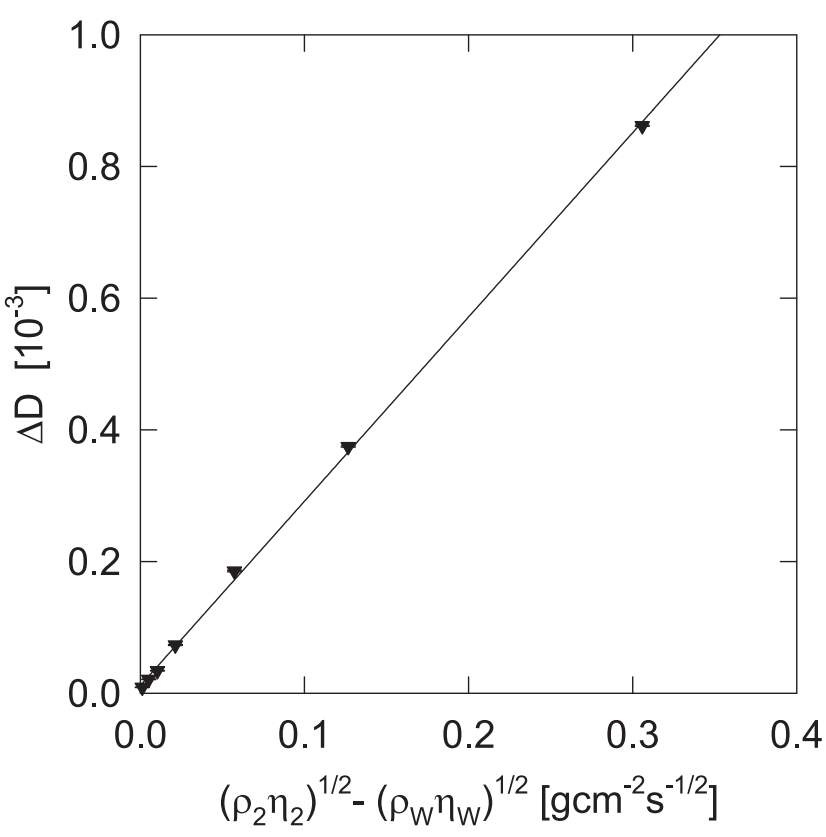

Fig. $4 \Delta D$ versus $\sqrt{\rho_{2} \eta_{2}}-\sqrt{\rho_{w} \eta_{w}}$ in PEG-6K solutions. The error bar represents the standard deviation. Measurements were repeated six times. The solid line shows the value calculated using a leastsquare method.

$\Delta D$ intercept is caused by the formation of a thin PEG layer on the gold electrode ${ }^{16,38)}$. The results of all the PEG solutions are summarized in Fig. 5 . The values of the $\Delta F$ slope rapidly decrease in the region from PEG-600 to PEG-500K. In contrast, the $\Delta D$ intercept is observed in all PEG solutions and has a constant value of $1.75 \times 10^{-5}$ above PEG$6 \mathrm{~K}$.

\section{DISCUSSION}

\subsection{Behavior of $\Delta D$ slope in PEG solutions}

Figure 5 shows that the values of the $\Delta D$ slope rapidly decrease in the region from PEG-600 to PEG-500K. This rapid variation arises from the dynamic properties of PEG molecules in the region of viscous penetration depth, generated at the solid-liquid interface oscillating at $9 \mathrm{MHz}^{16,38}$. We discuss this rapid variation from the point of view of the Debye process, which explains a single-time relaxation process of a molecule ${ }^{7)}$. In that process, the dynamical compliance, $J^{*}(\omega)$, is given by

$$
J^{*}(\omega)=J^{\prime}-i J^{\prime \prime}=\frac{\Delta J}{1+\omega^{2} \tau^{2}}-i \frac{\Delta J \omega \tau}{1+\omega^{2} \tau^{2}},
$$

where $\Delta J$ is the relaxation strength of the molecule, $\tau$ is the relaxation time of the molecule and $\omega$ is the angular frequency of the oscillating plate. This equation shows that the imaginary part has the maximum value at $\omega=1 / \tau$. In other words, the molecule has the maximum energy loss at 


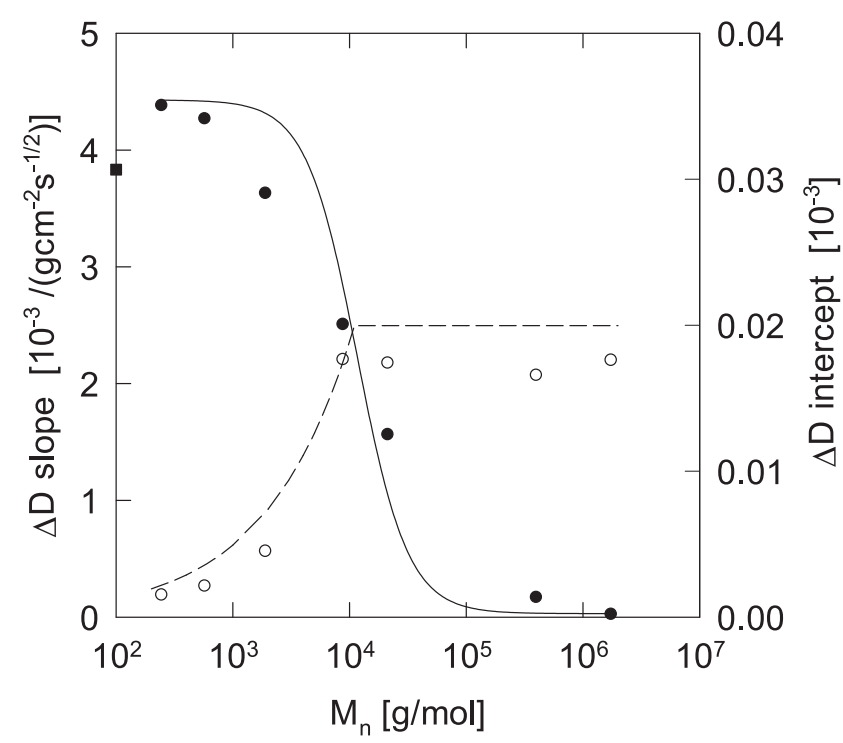

Fig. 5 Slope and intercept of $\Delta D$ versus $M_{\mathrm{n}}$ in PEG solutions. : $\Delta D$ slope, $\bigcirc: \Delta D$ intercept. The mark (ם) on $y$-axis means the slope value of equation (2). The solid line indicates the value calculated from real part of equation (3) using a nonlinear least-square method. The dashed line shows the value calculated from equations (4) and (12) with $\eta_{1}=\eta_{3}=8.90 \times 10^{-4} \mathrm{Ns} / \mathrm{m}^{2}, \rho_{1}=$ $\rho_{3}=997 \mathrm{~kg} / \mathrm{m}^{3}, \rho_{\mathrm{q}}=2648 \mathrm{~kg} / \mathrm{m}^{3}, h_{\mathrm{q}}=1.84 \times$ $10^{-4} \mathrm{~m}, \omega_{\mathrm{q}}=5.65 \times 10^{7} \mathrm{rad} / \mathrm{s}$ and $\mu_{3}=5.0 \times 10^{4}$ $\mathrm{N} / \mathrm{m}^{2}$, where $h_{3}$ is kept constant above $1.1 \times 10^{4}$ $\mathrm{g} / \mathrm{mol}$.

this point and resonates with the oscillating plate. Furthermore, the value of the real part is $\frac{\Delta J}{2}$ at this point. This means that $\frac{\Delta J}{2}$ is related to the molecular length resonating with the oscillating plate, i.e., the resonant length. Therefore, we adapt this concept to the present experimental data.

In our experiments, the $\Delta D$ slope corresponds to $\vec{J}$ of equation(3) and $M_{\mathrm{n}}$ is comparable to $\tau$ of equation(3). Therefore, we fitted the real part of equation (3) to the experimental values of the $\Delta D$ slope (Fig. 5). We found that the value of the $\Delta D$ slope corresponding to $\frac{\Delta J}{2}$ is $2.3 \times 10^{-3}$ $\left(\mathrm{gcm}^{-2} \mathrm{~s}^{-1 / 2}\right)^{-1}$.

Next, we investigated the relationship between $\frac{\Delta J}{2}$ and resonant length. From Fig. 5, the $M_{\mathrm{n}}$ value corresponding to $2.3 \times 10^{-3}$ is ca. $1.1 \times 10^{4} \mathrm{~g} / \mathrm{mol}$. Furthermore, the following relationship is proposed between molecular weight, $M$ and gyration radius, $R_{\mathrm{g}}^{13)}$ :

$$
R_{\mathrm{g}}=0.215 M^{0.583 \pm 0.031} .
$$

When $M_{\mathrm{n}}$ is considered as $M$, the $R_{\mathrm{g}}$ value obtained is 48.8 $\AA\left(=0.215 M^{0.583}\right)^{6,7,13)}$. That means that the PEG length of $97.6 \AA\left(=2 R_{\mathrm{g}}\right)$ moves with the oscillating QCM of $9 \mathrm{MHz}$.

\subsection{Behavior of $\Delta D$ intercept in PEG solutions}

The $\Delta D$ intercept arises from the thin layer of $\mathrm{PEG}$ formed by physical adsorption ${ }^{16,38)}$. From Fig. 3, we can see that the $\Delta D$ values vary linearly with $\sqrt{\rho_{2} \eta_{2}}-\sqrt{\rho_{w} \eta_{w}}$. This means that the properties of the thin PEG layer are independent of the bulk concentration over the range of concentrations tested. In addition, we can see from Fig. 5 that the values of the $\Delta D$ intercept increase with $M_{\mathrm{n}}$ and become constant above PEG-6K. We can exactly determine the molecular weight above which the $\Delta D$ intercept becomes constant.

To obtain further insight into the $\Delta D$ intercept, we discuss properties of the thin layer on the basis of the following equations proposed by Voinova et $a l .{ }^{16)}$ :

$$
\begin{aligned}
& \Delta f_{V}=\operatorname{Im}\left(\frac{\beta}{2 \pi \rho_{q} h_{q}}\right), \\
& \Delta D_{V}=-\operatorname{Re}\left(\frac{2 \beta}{\omega_{q} \rho_{q} h_{q}}\right), \\
& \beta=\kappa \xi \frac{1-A \exp \left(2 \xi h_{3}\right)}{1+A \exp \left(2 \xi h_{3}\right)}, \\
& A=\frac{\kappa \xi+\lambda \varsigma \tanh \left(\lambda h_{1}\right)}{\kappa \xi-\lambda \varsigma \tanh \left(\lambda h_{1}\right)} \\
& \kappa=\eta_{3}-i \frac{\mu_{3}}{\omega_{q}}, \xi=\sqrt{-\frac{\rho_{3} \omega_{q}^{2}}{\mu_{3}+i \omega_{q} \eta_{3}}} \\
& \lambda=\eta_{1}, \varsigma=\sqrt{\frac{i \rho_{1} \omega_{q}}{\eta_{1}}},
\end{aligned}
$$

where $\Delta f_{V}$ is the series resonant-frequency shift, $\Delta D_{V}$ is the shift in the energy dissipation factor, $h$ is the thickness and the subscript 3 indicates the thin layer. These equations describe properties of the one thin layer formed on the QCM in a Newtonian liquid, where the non-slip condition has been applied to the thin layer. Furthermore, the expansion of the $\beta$ expression in $\operatorname{Re}\left(\xi h_{3}\right)<<1, \operatorname{Im}\left(\xi h_{3}\right)<<1$ leads to the following equations:

$$
\begin{aligned}
& \Delta f_{V}=\frac{-1}{2 \pi \rho_{q} h_{q}}\left\{\frac{\eta_{1}}{\delta_{1}}+h_{3} \rho_{3} \omega_{q}-2 h_{3}\left(\frac{\eta_{1}}{\delta_{1}}\right)^{2} \frac{\eta_{3} \omega_{q}^{2}}{\mu_{3}^{2}+\omega_{q}^{2} \eta_{3}^{2}}\right\}, \\
& \Delta D_{V}=\frac{2}{\omega_{q} \rho_{q} h_{q}}\left\{\frac{\eta_{1}}{\delta_{1}}+2 h_{3}\left(\frac{\eta_{1}}{\delta_{1}}\right)^{2} \frac{\mu_{3} \omega_{q}}{\mu_{3}^{2}+\omega_{q}^{2} \eta_{3}^{2}}\right\}, \\
& \delta_{1}=\sqrt{\frac{2 \eta_{1}}{\rho_{1} \omega_{q}}},
\end{aligned}
$$

where $\delta_{1}$ is the penetration depth.

Equations (11) and (12) show that the physical properties of the $\Delta D_{V}$ intercept are clearly different from those of $\Delta f_{V}$. The $\Delta D_{V}$ intercept depends on the thickness, viscosity and elasticity of the thin layer, but not the density of the layer. If elasticity of the thin layer is zero, the $\Delta D_{V}$ intercept does not appear. Thus, the appearance of the $\Delta D$ intercept means that the thin PEG layer has a non-zero value of elas- 
ticity.

In our study, the values of the $\Delta D$ intercept are those at infinite dilution. In order words, the present experimental data of the $\Delta D$ intercept are of the thin layer immersed in pure water, which shows properties of a Newtonian liquid. In addition, the non-slip condition can be applied to the physical adsorption of PEG molecules onto the gold electrode at infinite dilution ${ }^{18}$. As a result, the present experimental data of the $\Delta D$ intercept can be discussed on the basis of equation (12).

Equation (12) shows that the $\Delta D_{V}$ intercept arises from the second term in relation to the thin layer. This further supports the idea that the $\Delta D$ intercept is caused by the thin PEG layer. Figure 5 shows that the values of the $\Delta D$ intercept become constant beyond the thickness of a PEG-6K layer. This result suggests that the variation in the $\Delta D$ intercept with $M_{\mathrm{n}}$ is mainly caused by the thickness of thin PEG layer. Therefore, equation(12) leads us to the assumption that the $\Delta D_{V}$ intercept in a PEG thin layer is mainly dependent on $h_{3}$, and the value of $\left(\frac{\eta_{1}}{\delta_{1}}\right)^{2} \frac{\mu_{3} \omega_{q}}{\mu_{3}^{2}+\omega_{q}^{2} \eta_{3}^{2}}$ is always independent of $M_{\mathrm{n}}$. This means the variations in density, viscosity and elasticity of the thin layer due to $M_{\mathrm{n}}$ are negligible.

Based on the above assumption, in the case of infinite dilution at $25^{\circ} \mathrm{C}\left(\eta_{1}=\eta_{3}=8.90 \times 10^{-4} \mathrm{Ns} / \mathrm{m}^{2}\right.$ and $\rho_{1}=\rho_{3}=$ $997 \mathrm{~kg} / \mathrm{m}^{3}$ ), we can estimate the value of elasticity of the thin layer to be a constant at $5.0 \times 10^{4} \mathrm{~N} / \mathrm{m}^{2}$. The variation of the $\Delta D_{V}$ intercept with $M_{\mathrm{n}}\left(h_{3}\right.$ calculated from equation (4)) is shown in Fig. 5. The theoretical value is consistent with the experimental data, where $h_{3}$ is set at a constant value, when $M_{\mathrm{n}}$ is greater than $1.1 \times 10^{4} \mathrm{~g} / \mathrm{mol}$.

This result supports the assumption that the variation in the $\Delta D_{V}$ intercept in a thin PEG layer with $M_{\mathrm{n}}$ is mainly caused by $\mathrm{h}_{3}$. In addition, we can observe that the $\Delta D$ intercept becomes constant when the molecular weight is above $1.1 \times 10^{4} \mathrm{~g} / \mathrm{mol}$. The value of $1.1 \times 10^{4} \mathrm{~g} / \mathrm{mol}$ is also equal to the resonant length of the $\Delta D$ slope. Thus, it can be derived that the thickness of the thin PEG layer above the resonant length does not affect the $\Delta D$ intercept.

In conclusion, we found that the main factor determining the variation in the $\Delta D$ intercept with $M_{\mathrm{n}}$ is the thickness of the thin layer and that the $\Delta D$ intercept becomes constant above the resonant length of the PEG molecule.

\subsection{Comparison between the resonant lengths of $\Delta D$ and $\Delta F$}

In the previous report ${ }^{37)}$, the series resonance-frequency shift, $\Delta F$, was used to estimate the resonant length to be $54.2 \AA$ in the regions of both the thin layer and the viscous penetration depth. On the other hand, in this work, the consideration of $\Delta D$ leads to a resonant length of $97.6 \AA$. The difference in the resonant length between $\Delta D$ and $\Delta F$ is $43.4 \AA$. In this section, we want to address this differ-

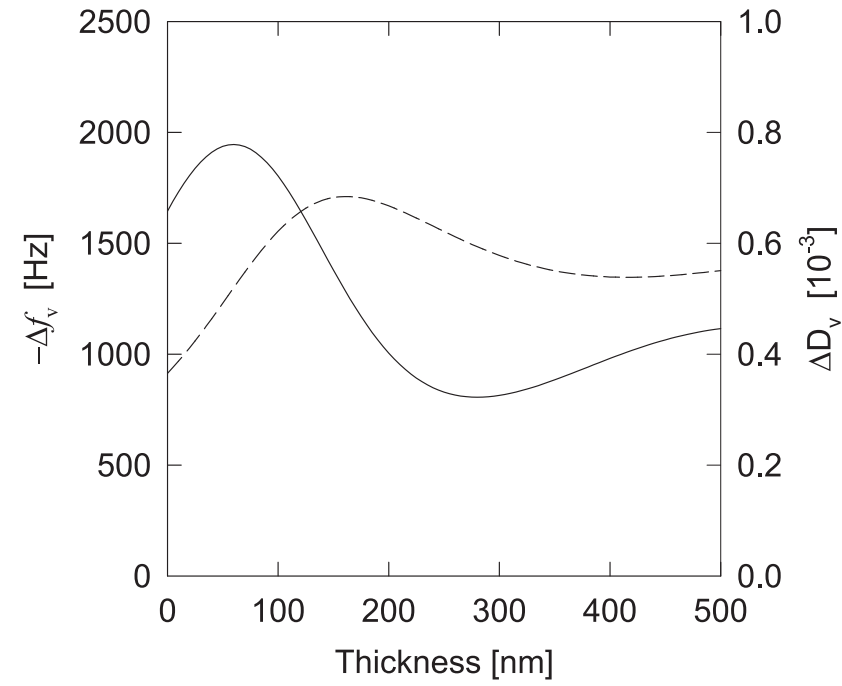

Fig. 6 Numerically calculated shift of $\Delta f_{v}$ and $\Delta D_{v}$ versus thickness of the thin layer. The solid line and the dashed line show the values calculated from equations (5) and (6) with $\eta_{1}=\eta_{3}=8.90 \times 10^{-4}$ $\mathrm{Ns} / \mathrm{m}^{2}, \rho_{1}=\rho_{3}=997 \mathrm{~kg} / \mathrm{m}^{3}, \rho_{\mathrm{q}}=2648 \mathrm{~kg} / \mathrm{m}^{3}$, $h_{\mathrm{q}}=1.84 \times 10^{-4} \mathrm{~m}, \omega_{\mathrm{q}}=5.65 \times 10^{7} \mathrm{rad} / \mathrm{s}$ and $\mu_{3}$ $=5.0 \times 10^{4} \mathrm{~N} / \mathrm{m}^{2}$. The solid line and the dashed line show $\Delta f_{\mathrm{v}}$ and $\Delta D_{\mathrm{v}}$, respectively.

ence.

Using equations (5) and (6), we calculated the values of $\Delta f_{V}$ and $\Delta D_{V}$ as a function of the thickness of the thin layer. The results are shown in Fig. 6. The thicknesses corresponding to the maximum values of $\Delta f_{V}$ and $\Delta D_{V}$ are different and $h_{f}<h_{D}$, where $h_{f}$ and $h_{D}$ are the thickness corresponding to the maximum values of $\Delta f_{V}$ and $\Delta D_{V}$, respectively. The values of $h_{f}$ and $h_{\mathrm{D}}$ correspond to the resonant lengths of PEG molecules. On comparing equations (11) and (12), it is obvious that this difference is based on the density of the thin layer.

In this work, we found that the density of the thin layer plays an important role in the dynamic properties of PEG at the solid-liquid interface, and that the resonant length is dependent on the measurement parameters.

\section{CONCLUSIONS}

We investigated the dynamic properties of PEG molecules on a QCM plate oscillating at $9 \mathrm{MHz}$ in terms of $\Delta D$. This study yielded the following results.

1 . The values of the $\Delta D$ slope against $\mathrm{M}_{\mathrm{n}}$ decrease according to the Debye process (equation (3)) and is caused by the effect of viscous penetration depth.

2 . The values of the $\Delta D$ intercept increase according to equation (12) and become constant above the resonant length. This behavior is caused by the formation of a 
thin layer of PEG on the QCM surface and is related to the length of the PEG molecule.

3. From the point of view of $\Delta D$, PEG of length $97.6 \AA$ moves with the oscillating plate at a frequency of $9 \mathrm{MHz}$ in the regions of the thin layer and viscous penetration depth.

4. The resonant length calculated using $\Delta D$ is larger than that calculated using $\Delta F$. This difference between the resonant lengths of $\Delta D$ and $\Delta F$ results from the density of the viscoelastic thin layer.

In conclusion, the behavior of PEG solutions clearly differs from that of a Newtonian liquid. Identifying the length of the polymer molecule moving with the QCM plate is very important to understanding the dynamic properties of polymer molecules at a solid-liquid interface.

\section{References}

1) Sauerbrey, G. Verwendung von Schwingquarzen zur Wägung dünner Schichten und zur Mikrovägung. $Z$. Physik. 155, 206-222 (1959).

2) Nomura, T. Application of Piezoelectric Quartz Crystals to Chemical Analysis in Liquids. Bunseki Kagaku 47, 751-767 (1998).

3) Ishii, R.; Nagasawa, R.; Nishioka, M.; Hanaoka, T. Microporous Organic-Inorganic Nanocomposites as the Receptor in the QCM Sensing of Toluene Vapors. Anal. Scie. 29, 283-289 (2013).

4) Steinem, C.; Janshoff, A. Piezoelectirc Sensors, Springer, New York, (2007).

5) Yoshimoto, M.; Shirahama, H.; Kurosawa, S.; Naito, M. Periodic Change of Viscosity and Density on Oscillating Chemical Reaction. J. Chem. Phys. 120, 70677070 (2004).

6) Teraoka, I. Polymer Solutions, John Wiley \& Sons, New York, (2002).

7) Strobl, G. The Physics of Polymers, Springer, New York, (2007).

8) Tanford, C. Physical Chemistry of Macromolecules, Wiley, New York, (1961).

9) Morawetz, H. High Polymers, 2nd ed.; Macromolecules in Solution, Wiley, New York, (1975).

10) De Gennes, P. G. Dynamics of Entangled Polymer Solutions. I. The Rouse Model. Macromolecules 9, 587593(1976).

11) De Gennes, P. G. Scaling Concepts in Polymer Physics, Cornell University Press, Ithaca, New York, (1979).

12) Hosek, M.; Tang, J. X. Polymer-Induced Bundling of F Actin and the Depletion Force. Phys. Rev. E 69, 051907-1-051907-10 (2004).

13) Devanand, K.; Selser, J. C. Asymptotic Behavior and Long-Range Interactions in Aqueous Solutions of Poly (ethylene oxide). Macromolecules 24, 5943-5947
(1991).

14) Mohsen-Nia, M.; Modarress, H.; Rasa, H. Measurement and Modeling of Density, Kinematic Viscosity, and Refractive Index for Poly (ethylene glycol) Aqueous Solution at Different Temperatures. J. Chem. Eng. Data 50, 1662-1666 (2005).

15) Noskov, B. A.; Akentiev, A. V.; Loglio, G.; Miller, R. Dynamic Surface Properties of Solutions of Poly (ethylene oxide) and Polyethylene Glycols. J. Phys. Chem. B 104, 7923-7931 (2000).

16) Voinova, M. V.; Rodahl, M.; Jonson, M.; Kasemo, B. Viscoelastic Acoustic Response of Layered Polymer Films at Fluid-Solid Interface: Continuum Mechanics Approach. Physica Scripta. 59, 391-396 (1999).

17) Wang, P.; Fang, J.; Qin, S.; Kang, Y.; Zhu, D.-M. Molecular Weight Dependence of Viscosity and Shear Modulus of Polyethylene Glycol(PEG) Solution Boundary Layers. J. Phys. Chem. C 113, 13793-13800 (2009).

18) Wang, P.; Fang, J.; Hou, Y.; Du, X.; Zhu, D.-M. Viscoelastic Properties of Polyethylene Glycol(PEG) Boundary Layers near a Solid Substrate. J. Phys. Chem. C 113, 729-735 (2009).

19) Höök, F.; Kasemo, B.; Nylander, T.; Fant, C.; Sott, K.; Elwing, H. Variations in Coupled Water, Viscoelastic Properties, and Film Thickness of a Mefp-1 Protein Film during Adsorption and Cross-Linking: A Quartz Crystal Microbalance with Dissipation Monitoring, Ellipsometry, and Surface Plasmon Resonance Study. Anal. Chem. 73, 5796-5804(2001).

20) Chirra, H. D.; Hilt, J. Z. Nanoscale Characterization of the Equilibrium and Kinetic Response of Hydrogel Structures. Langmuir 26, 11249-11257 (2010).

21) Brass, D. A.; Shull, K. R. Contact Studies of Weakly Compressed PEG Brushes with a Quartz Crystal Resonator. Langmuir 22, 9225-9233 (2006).

22) Harris, J. M. Poly (ethylene glycol)Chemistry, Biothechnical and Biomedical applications, Plenum Press, New York, (1992).

23) Powell, G. M. Polyethylene Glycol. In Handbook of Water Soluble Gums and Resins; Davidson, R. I., Ed., McGraw-Hill Book Company, New York, Chapter 18, (1980).

24) Elbert, D. L.; Hubbell, J. A. Surface Treatments of Polymers for Biocompatibility. Ann. Rev. Mater Sci. 26, 365-394 (1996).

25) Menz, B.; Knerr, R.; Göpferich, A.; Steinem, C. Impedance and QCM Analysis of the Protein Resistance of Self-Assembled PEGylated Alkanethiol Layers on Gold. Biomaterials 26, 4237-4243 (2005).

26) Hoffmann, C.; Tovar, G. E. M. Mixed Self-Assembled Monolayers (SAMs) Consisting of Methoxy-Tri (ethylene glycol)-Terminated and Alkyl-Terminated Dimethylchlorosilanes Control the Non-specific Adsorption of Proteins at Oxidic Surfaces. J. Colloid Interface Sci. 
295, 427-435(2006).

27) Michel, R.; Reviakine, I.; Sutherland, D.; Fokas, C.; Csucs, G..; Danuser, G.; Spencer, N. D.; Textror, M. A Novel Approach to Produce Biologically Relevant Chemical Patterns at the Nanometer Scale: Selective Molecular Assembly Patterning Combined with Colloidal Lithography. Langmuir 18, 8580-8586 (2002).

28) Unsworth, L. D.; Sheardown, H.; Brash, J. L. Protein Resistance of Surfaces Prepared by Sorption of EndThiolated Poly (ethylene glycol) to Gold: Effect of Surface Chain Density. Langmuir 21, 1036-1041 (2005).

29) Satomi, T.; Nagasaki, Y.; Kobayashi, H.; Tateishi, T.; Kataoka, K.; Otsuka, H. Physicochemical Characterization of Densely Packed Poly (ethylene glycol) Layer for Minimizing Nonspecific Protein Adsorption. J. Nanosci. Nanotechnol 7, 2394-2399 (2007).

30) Heuberger, M.; Drobek, T.; Spencer, N. D. Interaction Forces and Morphology of a Protein-Resistant Poly (ethylene glycol) Layer. Biophys. J. 88, 495-504 (2005).

31）Kizhakkedathu, J. N.; Janzen, J.; Le, Y.; Kainthan, R. K.; Brooks, D. E. Poly (oligo (ethylene glycol) acrylamide) Brushes by Surface Initiated Polymerization: Effect of Macromonomer Chain Length on Brush Growth and Protein Adsorption from Blood Plasma. Langmuir 25, 3794-3801 (2009).

32) Reimhult, K.; Petersson, K.; Krozer, A. QCM-D Analysis of the Performance of Blocking Agents on Gold and Polystyrene Surfaces. Langmuir 24, 8695-8700 (2008).

33) Wei, J.; Ravn, D. B.; Gram, L.; Kingshott, P. Stainless Steel Modified with Poly (ethylene glycol)can Prevent
Protein Adsorption but not Bacterial Adhesion. Colloids Surf. B. Biointerfaces 32, 275-291 (2005).

34) Yoshikawa, C.; Goto, A.; Tsujii, Y.; Fukuda, T.; Kimura, T.; Yamamoto, K.; Kishida, A. Protein Repellency of Well-Defined, Concentrated Poly (2-hydroxyethyl methacrylate) Brushes by the Size-Exclusion Effect. Macromolecules 39, 2284-2290(2006).

35) Notley, S. M. Adsorption of Polyelectrolyte Modified Graphene to Silica Surfaces: Monolayers and Multilayers. J. Colloid Interface Sci. 375, 35-40 (2012).

36) Marcus, I. M.; Herzberg, M.; Walker, S. L.; Freger, V. Pseudomonas Aeruginosa Attachment on QCM-D Sensors: The Role of Cell and Surface Hydrophobicities. Langmuir 28, 6396-6402 (2012).

37) Yoshimoto, M.; Yuda, Y.; Aizawa, H.; Sato, H.; Kurosawa, S. Dynamic Properties of the Polyethylene Glycol Molecules on the Oscillating Solid-Liquid Interface. Anal. Chim. Acta 731, 82-87(2012).

38) Buttry, D. A.; Ward, M. D. Measurement OF Interfacial Processes at Electrode Surfaces with the Electrochemical Quartz Crystal Microbalance. Chem. Rev. 92, 1355-1379 (1992).

39) Arnau, A. Piezoelectric Transducers and Applications, Springer, New York, (2008).

40) Kanazawa, K. K.; Gordon, J. G. the Oscillation Frequency of a Quartz Resonator in Contact with a Liquid. Anal. Chim. Acta 175, 99-105(1985).

41) Rodahl, M.; Höök, F.; Kasemo, B. QCM Operation in Liquids: An Explanation of Measured Variations in Frequency and Q Factor with Liquid Conductivity. Anal. Chem. 68, 2219-2227(1996). 